\title{
A low-complexity energy disaggregation method: Performance and robustness
}

\author{
Hana Altrabalsi, Jing Liao, Lina Stankovic and Vladimir Stankovic \\ Department of Electronic and Electrical Engineering \\ University of Strathclyde, Glasgow, G1 1XW, UK \\ Email: \{h.altrabalsi, jing.liao, lina.stankovic, vladimir.stankovic\}@strath.ac.uk.
}

\begin{abstract}
Disaggregating total household's energy data down to individual appliances via non-intrusive appliance load monitoring (NALM) has generated renewed interest with ongoing large-scale smart meter deployments. Of special interest are NALM algorithms that are of low complexity and operate in real time, supporting emerging applications such as remote appliance scheduling and home automation, and use low sampling rates data from commercial smart meters. NALM methods, based on Hidden Markov Model (HMM) and its variations, have become the state of the art due to their high performance, but suffer from high computational cost. In this paper, we develop an alternative approach based on support vector machine (SVM) and k-means, where k-means is used to reduce the SVM training set size by identifying only the representative subset of the original dataset for the SVM training. The resulting scheme outperforms individual k-means and SVM classifiers and shows competitive performance to the state-of-the-art HMM-based NALM method with up to 45 times lower execution time.
\end{abstract}

\section{INTRODUCTION}

A large scale deployment of smart meters in households has started or it is about to start in many countries worldwide. For example, the UK Government has committed utilities to a roll out of automatic meter reading (AMR) systems by 2020. It is anticipated that by 2020 all UK households will be equipped with an AMR system that measures and displays in real time aggregate energy usage with an in-home display unit [1]. This large governmental investment promises significant improvements in energy demand via automatic, more efficient and more informed billing.

While the proposed acquisition, control, and communications technologies have already been developed and agreed [1], it is still not clear how the massive amount of collected smart meter data will be utilized to ensure consumer-tailored and timely energy saving advice. One attractive solution is to provide appliance-itemized billing, which requires monitoring individual appliances, and could be more informative than current billing practices.

Since it is impractical to install and maintain individual load sensors for each and every appliance in a household, non-intrusive appliance load monitoring (NALM), i.e., disaggregating individual appliance usage from the total, aggregated energy consumption captured at the energy monitor, is becoming increasingly popular. Besides appliance-itemized billing, NALM is useful to customers to determine which appliances are the most energy consuming ones, which are faulty, and when it is time to replace or service an old appliance. NALM is also beneficial to suppliers to better plan power demand, to system operators to monitor the effect of smart grid fluctuations on the residential microgrid, to appliance manufacturers and policy makers.

NALM appeared in the research literature in 1980's [2], and since then, many NALM algorithms have been proposed that improve the initial design of [2] and adapt to advances in sensor technology capturing energy measurands at a range of sampling rates, generally in the order of $\mathrm{kHz}$. However, with large-scale smart metering deployment on the way, the increased interest is in NALM algorithms that work at lower sampling rates, in the order of seconds and minutes. It is not only the cost of the sensing technology [3], but also computational and storage cost as well as implementation efficiency that are key drivers towards the wide deployment of low-sampling smart meters. However, so far, there are no widely available efficient solutions for NALM, that offer high accuracy and low complexity at low sampling rates [4], [5].

Conventional event-based NALM algorithms consist of four steps. First, signal pre-processing is carried out, usually via data cleaning and filtering. Then, event detection is performed to isolate events when the state of an appliance has changed. After event detection, feature extraction is applied to isolate power features or signatures from the identified events, and finally, a classification method is used to classify extracted features into appliance groups. Many techniques have been applied to perform classification and optimization to design classifiers such as fuzzy logic, Naive Bayes, kNN, k-means, mean-shift, decision trees, neural networks, support vector machine, Hidden Markov Model, and many hybrid approaches (see [4]-[6] and references there in).

Recently, state-based approaches that model devices using a small number of appliance states (e.g., on, off, standby state), such as Hidden Markov Model (HMM) and its variations, have become popular for NALM, because they are good at modelling the combination of stationary processes, with continuous valued data over discrete time (see [5], [7]-[10] and references there in). HMM can probabilistically model sequential data, incorporating, in the learning process, the timedependency in running appliances as well as the transition of the appliance through different states during its operation. According to [5], state-of-the-art HMM-based methods [8], [10], [11] work offline, can be supervised or unsupervised, and are not scalable. Furthermore, the complexity of HMM 
exponentially increases with the number of appliances, and the whole model needs to be retrained when a new appliance is added [5].

Another popular technique used for NALM is Support Vector Machine (SVM). SVM-based NALM has shown good performance especially for low frequency load features [5], it is scalable, and is a well established method for classifying noisy data. Non-linear classifiers, such as kernel SVM, that map the input feature space into a high dimensional space and find the optimal separating hyperplane between two classes to separate them, is one of the most effective classification methods, but has at least quadratic training time complexity. Thus, similarly to HMM, SVM-based approaches suffer from high computational complexity, due to necessary training on large scale data, which makes them unsuitable for real-time NALM applications.

The key problem of HMM-based and SVM-based approaches is their high computational cost, that prevents their application for services that require real-time disaggregation, such as device scheduling, virtual power sensing, demand response capacity estimation, etc [6]. Motivated by increased demands for real-time NALM, we develop and analyze low-rate, low-complexity NALM methods paying particular attention to their applicability, in terms of running time, implementation issues and robustness to the size of the training set and training set labeling errors.

In particular, to benefit from high classification performance of non-linear SVMs and low computational cost of k-means clustering, we effectively combine conventional k-means and SVM obtaining a hybrid method that outperforms k-means and SVM classification alone. Inspired by [12]-[14], where $\mathrm{k}$-means and SVMs are combined to reduce complexity, we use k-means to cleverly select a subset of input data used to train a linear SVM. By training the SVM only on a small set of representative samples, we are able to significantly reduce training and testing computational cost while demonstrating similar performance to that of the state-of-the-art HMM-based NALM approaches, which is demonstrated in the Section IV for $1 \mathrm{~min}$ sampling rate and active power measurements only. The key contribution of this paper is:

- Novel real-time combined kmeans-SVM-based NALM method for low sampling rate data;

- Innovative appliance-based selection of extracted features that maximize performance;

- Experimental evaluation using different training sizes and errors in labeling the training data;

- Comparison with state-of-the-art approaches using three US households from the REDD data set [15].

The rest of the paper is organized as follows. Section II brings a brief background on NALM. Section III describes the proposed NALM algorithm. The last two sections discuss the simulation results, conclusion and future work.

\section{BACKGROUND AND LITERATURE REVIEW}

Non-Intrusive Appliance Load Monitoring (NALM), also referred to as NILM or NIALM [2], disaggregates total power readings and identifies each appliance in use at any point in time based on the available measured total household consumption.

Traditional NALM methods consist of signal preprocessing, edge detection and feature extraction followed by classification. After acquisition, signal pre-processing can be done in the form of power normalization, filtering (for signal smoothing and getting rid of sudden peaks), and thresholding to remove small power loads that would appear as noise as well as the base-load, from appliances that are always running. Next, edge detection is done to identify events of appliances switching on and off. Edge detection is followed by extracting the features in the identified event windows. Classification is then used to group sets of extracted windows which have similar characteristics, such as power levels, time profile, reactive components etc.

In this paper, we focus on low complexity, low-rate NALM algorithms, where sampling rates are in the range of seconds and minutes. The sampling rate influences the type of features that can be used. For example, low-rate NALM approaches can use only steady-state parameters, such as active or real power [9], reactive power [2], [4], power factor [16], voltage or current waveform [17], [18].

The simplest approach, from an implementation point of view, is to use a current transformer (CT) sensor attached to the wire via a clump to measure alternating current and an ACto-AC power adaptor with a circuit to measure voltage. This way, active and reactive power components can be calculated from the measured current and voltage. However, measuring voltage in a simple way requires additional plug points, which are often not available close to the electricity meter. Moreover, processing, communicating and storing two dimensional data (active and reactive power) is often impractical, especially because the reactive component is not needed for billing purposes. That is why, in this paper, we consider disaggregation using only active power values, obtained, for example, from the electric current measured via a simple CT sensor.

Based on the employed classification method, all NALM algorithms can be classified as supervised and non-supervised. Supervised NALM methods use labeled appliance events to train classifiers, and are usually based on optimization and pattern recognition approaches, such as rule-based, SVM or Bayes-based classification. Unsupervised methods do not require labeled sets and are usually based on clustering [19] or HMMs [8]-[10].

Early NALM work focused on disaggregation of high loads. In 1997, low-cost NALM technologies were prototyped by the Electric Power Research Institute (EPRI) [20] in collaboration with several utilities. The device was able to disaggregate on-off loads with $90 \%$ accuracy, refrigerators with $\sim 80 \%$, and failed to provide good results for multi-state appliances (such as washing machine, dishwasher, heat pumps, etc). Key obstacles towards massive NALM deployment were identified as a restricted set of traceable appliances (low loads cannot be detected), high cost, and high complexity for tracing multistate appliances. The HELP system of Powers et al. [21] 
disaggregates large loads such as air conditioners using very low granularity measurements, sampled every $15 \mathrm{mins}$, and extracting features such as occurrence, timing and magnitude of all large changes. Other NALM work for disaggregating large loads include [22], [23], and [24].

Recent work on NALM is mainly focused on state-based probabilistic methods. In [8] four different methods for lowrate NALM are proposed using (conditional) factorial HMM and Hidden semi-Markov models. The obtained accuracy was in the range between $72 \%$ and $99 \%$ for $3 \mathrm{sec}$ sampling rate in seven different houses with up to 10 appliances with an average accuracy of $83 \%$. This method cannot disaggregate base load, such as, for example, refrigerator, it is not of low computational complexity, and is prone to converge to a local minimum.

In [9] a factorial HMM is used for disaggregation of active power load at $1 \mathrm{~min}$ sampling rate. The method uses expert knowledge to set initial models for states of known appliances. To obtain reliable results, it is necessary to correctly set the a priori-values for each state for each appliance, which in turn is strongly dependent on the particular aggregate dataset on which NALM is being performed. Indeed, a similar factorial HMM-based approach is tested in [15], where it is shown that the disaggregation accuracy drops by up to $25 \%$ when different houses are used to set the initial models compared to the case when the same house is used for building the models (training) and testing. Results are reported for REDD dataset [15] with sampling rates of $1 \mathrm{sec}$ and $3 \mathrm{sec}$.

In [19], [25], and [26] a decision-tree (DT) classifier is used for pattern matching. The DT-based algorithm developed in [25], is a low-complexity, supervised approach that uses only rising and falling active power edges to build a DT model that is used for classification. The method is not scalable, since retraining is needed whenever a new appliance is added, but is fast and performs well even when the training period is very short.

In [10], an unsupervised Additive Factorial Approximate Maximum A-Posteriori (AFMAP) inference algorithm is proposed using differential factorial HMMs. First, all snippets of active power data are extracted using a threshold and modelled by an HMM; next the k-nearest-neighbor graph is used to build nine motifs that are treated as HMMs over which AFMAP is run. The results show average accuracy of $87.2 \%$ using 7 appliances and sampling rate of $60 \mathrm{~Hz}$. In [11] Hierarchical Dirichlet Process Hidden Semi-Markov Model (HDP-HSMM) factorial structure is used removing some limitations of the approach of [8] at increased complexity. The results are reported for five devices using 20sec resolution with 18 24hour segments across four houses from the REDD dataset [15] obtaining disaggregation accuracy of $81 \%$ outperforming the EM-based method of [15].

The main problem with the above state-based approaches is that they are not suitable for real-time applications due to their high computational complexity. See, for example, [6] for some examples. The low-complexity HMM-based method proposed in [27] reduced execution time 72.7 times, but still requires
11.4 seconds for disaggregating two appliances using 524,544 readings or 94 minutes for 11 appliances.

\section{PRoposed LOW-COMPLEXITY NALM}

In this section, we describe the proposed NALM method. First, we discuss pre-classification steps. Then, we show that trained k-means and SVM-based classification alone are not suitable for real-time NALM application, either due to modest performance or high execution time. Finally, we propose a combined k-means/SVM classification method using different extracted power features.

The algorithm comprises two phases: a training phase and a testing phase. Training is always done on aggregate data using a labeled dataset, which is obtained from time-diaries or submetering. The entire disaggregation procedure includes three steps: event detection, feature extraction, and classification. In the proposed solution we use the efficient edge detection method of [25] and focus on improving the feature extraction and classification steps. In the following, we closely follow the notion of [25], which we review next.

Let $\mathbf{M}$ be a set of all known appliances in the house. Let $p\left(t_{i}\right)$ be active power measured at time instance $t_{i}$. Without loss of generality, in the following we denote $p\left(t_{i}\right)$ as $p\left(t_{i}\right)=$ $p(i T)=p(i)$, where $T=t_{i}-t_{i-1}$ is the sampling interval.

The disaggregation task is to find $p_{j}(i)$ for all $j$, such that $p(i)=\sum_{j=1}^{M} p_{j}(i)+n(i)$, where $p_{j}(i) \geq 0$ is the power load of appliance $j$ and $n(i)$ is the measurement noise. Note that $p_{j}(i)$ is zero if the appliance is off at time instance $i T$.

\section{A. Event Detection and Feature Extraction}

The task of the event detection is to detect changes in timeseries aggregate load curve due to one or more appliance being switched on/off or changing its state. Let $W$ be a set threshold. Then, if $\left|p_{j}(i)-p_{j}(i-1)\right| \geq W$ then the appliance $j$ has changed state at time instant $i T$.

Threshold $W$ needs to be set low enough so that for all $j$, if $\left|p_{j}(i)-p_{j}(i-1)\right| \leq W$ Appliance $j$ did not change its state and, otherwise, it did change its state. $W$ depends on the set of appliances being monitored, and is adapted automatically during the training process based on the minimum state transition that needs to be detected and the maximum variation of the active power within one appliance state across all appliances' states, that is

$$
W=\max \left\{\min _{m \in \mathbf{M}} \mathbf{p}_{\mathbf{m}}, \max _{\mathbf{m} \in \mathbf{M}}\left|\max \left(\mathbf{p}_{\mathbf{m}}\right)-\min \left(\mathbf{p}_{\mathbf{m}}\right)\right|\right\},
$$

where $\mathbf{p}_{\mathbf{m}}$ is a vector of active power readings of appliance $m$. Note that the value of $W$ depends on the set of available appliances, and is adaptively changed as appliances are being disaggregated and removed from the aggregate load.

An event occurs whenever an appliance changes its state. Edge detection is used to detect events by comparing $\mid p(i)-$ $p(i-1) \mid$ with $W$. We say a window of the event started at time $l_{s}$ and ended at $l_{e}$ if an appliance changed its state at $l_{s}$ and $l_{e}$, and

$$
\left|\left[p\left(l_{s}\right)-p\left(l_{s}-1\right)\right]+\left[p\left(l_{e}\right)-p\left(l_{e}-1\right)\right]\right| \leq C,
$$


where $C$ is parameter smaller than $W$.

Next, from each detected event window, features are extracted and stored. Extracted features could be simply all active power readings in the event window, or only rising/falling edge, or maximum/minimum value, area, etc.

\section{B. Classification}

Extracted features from each detected event are matched to the pre-defined appliance classes using a trained classifier. First, we test two conventional techniques to perform classification and pattern matching: trained k-means and SVM.

Trained k-means uses the labeled dataset to find the optimal cluster heads and cluster distribution. The number of clusters is always set to the number of known appliances in the household. The aggregate load is then grouped into known appliance subsets and the centroid of each subset is set as cluster head. When a new testing sample (feature vector active power load) is introduced, it is compared to all cluster heads, and the minimum distance determines the classification outcome.

SVM-based algorithms are optimal classifiers in the presence of noise and proven to perform well for NALM applications [5]. We train binary classifiers to separate one appliance at the time. After an appliance has been classified, its contribution is removed, the threshold $W$ in (1) is adapted, and the next appliance is considered.

As will be shown in the next section, while the trained $\mathrm{k}$-means-based NALM is time efficient, it provides low disaggregation performance. On the other hand, the SVM-based NALM method significantly outperforms the trained k-meansbased approach, but requires up to 10 times more computational time.

In order to design a high-performance, low-complexity solution, we propose to use a linear SVM on a substantially reduced training set obtained using k-means. Combining kmeans and SVM has been studied before, but not in the context of NALM. Recognizing that in a majority of cases a large portion of the input data is redundant for training, in [28], $\mathrm{k}$-means is used to decrease the number of support vectors and the training set size. Similarly, in [12], [13], k-means is employed to select a subset of original data for the SVM training. In [14], a clustered SVM is proposed that, in a divideand-conquer manner, trains a linear SVM on each of the kmeans clusters.

To combine k-means and SVM, we first train k-means as explained above using the entire original dataset. As a result, $k$ clusters each corresponding to one appliance are formed with a centroid as cluster head. All feature vectors falling in Cluster $i$ that are at an Euclidian distance larger than $r$ from their cluster head, form a subset $C_{i}$ that is used to train a linear SVM for Appliance $i . r$ is a pre-set threshold, obtained heuristically, that is used to tradeoff complexity and performance. Algorithm 1 shows the training steps, where $d(x, y)$ denotes the Euclidian distance between vectors $x$ and $y$.

During testing, if the Euclidian distance between a tested sample and any cluster head is smaller than a pre-set threshold,

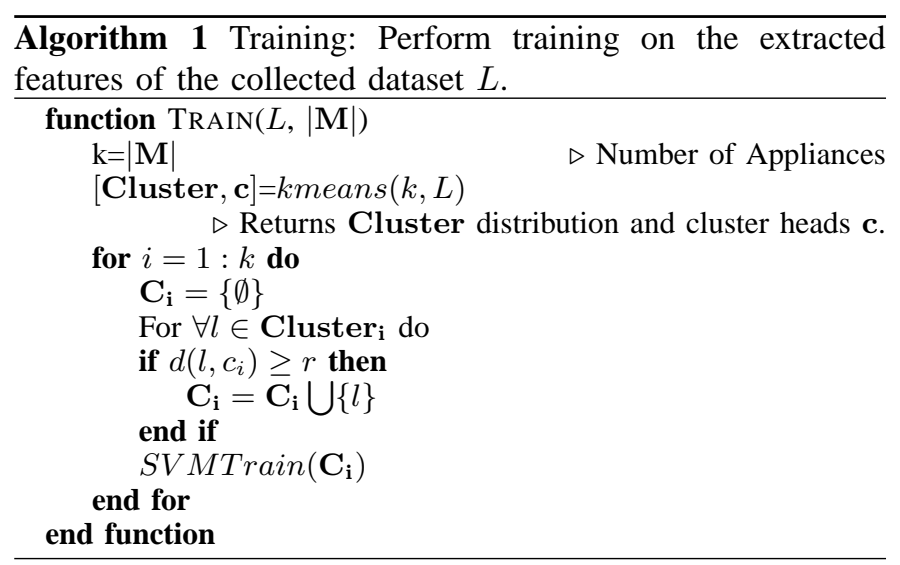

then the sample is classified to the closest cluster head using the trained k-means. Otherwise, the sample is input to the SVM classifier. The proposed combined method has low execution time, since many samples are going to be classified rapidly using k-means, and has good performance, since SVM improves classification for samples that would most likely be incorrectly classified using the trained k-means.

\section{RESUlTS AND Discussion}

In this section we present our experimental results and discuss our main findings. We use House 1, House 2 and House 6 from the publicly available REDD database [15] downsampled to $1 \mathrm{~min}$ resolution. The training size was varied in the experiments, and testing is always performed on four weeks worth of data.

The evaluation metrics used are precision (PR), recall (RE) and F-Measure $\left(F_{M}\right)$ [29] defined as:

$$
\begin{gathered}
P R=T P /(T P+F P) \\
R E=T P /(T P+F N) \\
F_{M}=2 *(P R * R E) /(P R+R E),
\end{gathered}
$$

where true positive (TP) presents the correct claim the detected event was triggered by the use of the appliance, false positive (FP) represents an incorrect claim that an appliance triggered the detected event, and false negative $(\mathrm{FN})$ indicates that the appliance used was not identified.

Table I shows results obtained using House 1 data for the trained k-means-based, SVM-based, and the combined algorithm. All three algorithms always use the same edge detection and feature extraction method explained in the previous section. In House 1, we trained the algorithms with the following five known appliances: refrigerator, washer dryer, dish washer, toaster, and microwave. All other household appliances were considered to be unknown and hence they contribute to noise $n(i)$. The training size contains 7000 samples or roughly one week of data. All experiments were run on an HP Pavilion 15 Notebook PC with 8GB RAM, 1TB Hard drive and AMD A10 with $2.2 \mathrm{GHz}$ Radeon HD dual Graphics processor (quad core). We tested different two-, three-, four, five-dimensional classifiers by extracting different features and present results 
TABLE I

COMPARISON BETWEen THE THREE METHODS USING $F_{M}$ AND EXECUTION TIME USING REDD House 1.

\begin{tabular}{|c|c|c|c|c|c|c|c|c|c|}
\hline & \multicolumn{3}{|c|}{$S V M$} & \multicolumn{3}{|c|}{$k$-means } & \multicolumn{3}{|c|}{ Combined method } \\
\hline Features & $\operatorname{train}(\mathrm{sec})$ & test $(\mathrm{sec})$ & $F_{M}(\%)$ & $\operatorname{train}(\mathrm{sec})$ & test $(\mathrm{sec})$ & $F_{M}(\%)$ & $\operatorname{train}(\mathrm{sec})$ & test $(\mathrm{sec})$ & $F_{M}(\%)$ \\
\hline Max and duration & 0.74 & 0.69 & 71.3 & 0.17 & 0.17 & 70.6 & 0.36 & 0.10 & 75.94 \\
\hline Max and Max/mean & 0.82 & 0.60 & 76.1 & 0.17 & 0.17 & 70.2 & 0.32 & 0.05 & 73.1 \\
\hline Max, area and Max/mean & 1.12 & 0.67 & 70.7 & 0.26 & 0.26 & 65.1 & 0.36 & 0.45 & 53.3 \\
\hline Min, area and Max/mean & 1.29 & 0.7 & 68.8 & 0.19 & 0.19 & 68.7 & 0.56 & 0.41 & 69.6 \\
\hline
\end{tabular}

for the best two 2D and two 3D classifiers. The results are averaged over all known appliances.

It can be seen from Table I that the SVM-based method always outperforms the trained k-means, but requires more time for both training and testing. The best SVM-based NALM result is obtained for the $2 \mathrm{D}$ classifier using maximum and maximum/mean factor and performs $6 \%$ better than the best $\mathrm{k}$-means-based performance, but is more than 4 and 3 times slower when performing training and testing, respectively. The combined approach provides a good tradeoff reducing the training and testing time by over 2 and 6 times, respectively, compared to the SVM-based method.

Interestingly, the selection of features has a significant impact on the performance. For example, using an area as a feature together with maximum/mean factor and maximum or minimum does not lead to good results. The best combined method is only $0.15 \%$ worse than the best SVM-based approach, but reduces the execution time by over three times.

Table II shows results per appliance for the combined method. Marked with bold typeface are the best features for each appliance. All features denote the 5D case, where max, min, max/mean, duration and area are used. One can see that for different appliances different features give the best performance. For example, only 5D classification gives nonzero disaggregation for the toaster. Since we are classifying one appliance at a time, it is possible to adapt classification features from appliance to appliance. Thus, during the training, the best features to use are identified per appliance which are then used during testing. In the following, we refer to this method, as the proposed combined method.

Table III shows the obtained results for all known appliances in House 2. In House 2, there are five known appliances listed in the first column of the table. All other appliances are considered unknown. We compare the proposed approach with the state-of-the art HMM-based method of [9], which was designed for low-sampling (1 $\mathrm{min})$ rates. For each dataset, all three tested algorithms always use the same amount of data for training (7000 samples or roughly one week) and testing (four weeks). The HMM-based method [9] requires prior initialization of the model using expert knowledge (state variances, mean value for each state and state transition probabilities), which was carried out in our experiments either using the information provided by the authors of [9], or were

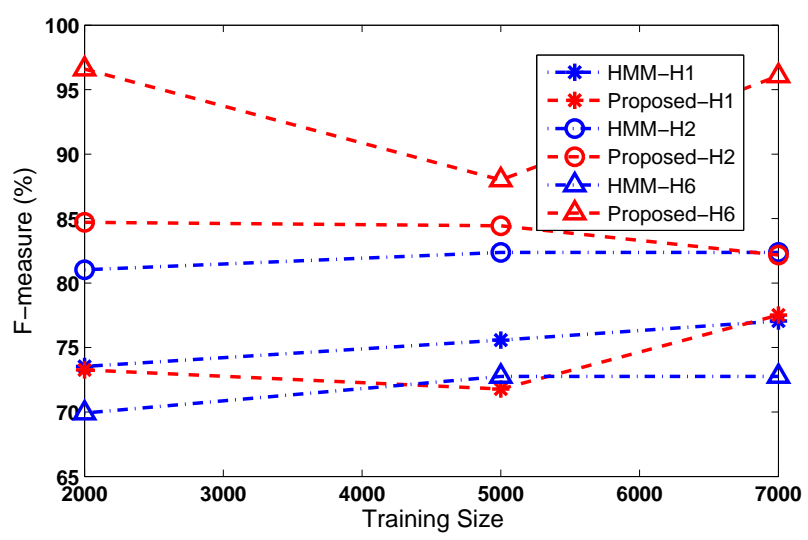

Fig. 1. Effect of varying the training size on the performance of the two NALM methods for the three REDD houses.

generated during training. The SVM-based method selects the best features to use for each appliance and then performs SVM-based classification.

It can be seen from the table that the proposed method outperforms the HMM-based approach for all appliances except the stove, which was often confused for the refrigerator, and microwave, for which SVM shows poor result. The proposed algorithm is always better than the SVM-based approach. All three algorithms struggle with the dish washer, but show very good results for the refrigerator, which was always on, so the number of event samples for training was the largest.

Table IV shows the average results for all three tested houses. It can be seen that the proposed method provides the same or better performance than HMM, while its execution time (including training and testing) is up to 240 times less. The SVM-based method requires more time than the proposed method and provides similar performance.

Next, we test the robustness of the proposed methods to the reduction of the training set size. Tables V, VI and Figure 1 show the execution time (for training and for testing) and $F_{M}$ results, respectively, for 3 different training sizes, with 2000 (roughly 2 days), 5000 (roughly 5 days), and 7000 samples. It can be seen that the methods are robust to the variation of the training size. The HMM-based method only requires one appliance event running alone to build a model. If the 
TABLE II

$F_{M}$ RESULTS FOR THE COMBINED METHOD FOR DIFFERENT APPLIANCES AND DIFFERENT EXTRACTED FEATURES USING REDD HOUSE 1.

\begin{tabular}{|c||c|c|c|c|c|}
\hline \multicolumn{1}{|c||}{ Features } & \multicolumn{5}{|c|}{$F_{M}(\%)$} \\
\hline \hline Max and area & $\mathbf{8 9 . 4 9}$ & 20.7 & 0 & 28.7 & 53.7 \\
\hline Max, Min and Max/mean & 85.68 & $\mathbf{6 3}$ & 0 & 7.27 & 40.75 \\
\hline All features & 84.51 & 15.38 & $\mathbf{4 . 7 6}$ & 11.76 & 57.53 \\
\hline Min and Max/mean & 83.35 & 16.21 & 0 & $\mathbf{3 5 . 9 7}$ & 29.16 \\
\hline Max and Duration & 88.51 & 56 & 0 & 32.5 & $\mathbf{7 5 . 3 6}$ \\
\hline
\end{tabular}

TABLE III

COMPARISON BETWEEN THE THREE NALM METHODS USING REDD HOUSE 2.

\begin{tabular}{|c||c|c|c||c|c|c||c|c|c|}
\hline \multicolumn{1}{|c||}{} & \multicolumn{3}{c|}{$P R(\%)$} & \multicolumn{3}{c||}{$R E(\%)$} & \multicolumn{3}{c|}{$F_{M}(\%)$} \\
\hline Appliances & $H M M$ & $S V M$ & Proposed & $H M M$ & $S V M$ & Proposed & $H M M$ & $S V M$ & Proposed \\
\hline \hline Refrigerator & 87.45 & 93.41 & 92.87 & 87.93 & 91.76 & 95.88 & 87.69 & 92.58 & 94.35 \\
\hline Stove & 38.10 & 4.1 & 66.6 & 66.67 & 33.3 & 33.3 & 48.48 & 7.4 & 44.4 \\
\hline Microwave & 35.71 & 0 & 15.38 & 58.14 & 0 & 82.05 & 44.25 & 0 & 25.91 \\
\hline Toaster & 54.9 & 54.9 & 69.6 & 92.45 & 93.3 & 91.66 & 64.90 & 69.1 & 79.13 \\
\hline Dish Washer & 33.33 & 33.3 & 22.22 & 7.56 & 21.42 & 42.85 & 12.32 & 26.08 & 29.2 \\
\hline
\end{tabular}

TABLE IV

COMPARISON BETWEEN THE THREE NALM METHODS FOR THE THREE REDD HOUSES. ALL RESULTS ARE AVERAGED OVER ALL KNOWN APPLIANCES.

\begin{tabular}{|c|c|c|c|c|c|c|c|c|c|c|c|c|}
\hline & \multicolumn{4}{|c|}{$H M M$} & \multicolumn{4}{|c|}{$S V M$} & \multicolumn{4}{|c|}{ Proposed method } \\
\hline House & $P R(\%)$ & $R E(\%)$ & $F_{M}(\%)$ & Time $(\mathrm{sec})$ & $P R(\%)$ & $R E(\%)$ & $F_{M}(\%)$ & Time $(s e c)$ & $P R(\%)$ & $R E(\%)$ & $F_{M}(\%)$ & Time $(\mathrm{sec})$ \\
\hline House 1 & 77.16 & 76.97 & 77.06 & 51.22 & 80.3 & 80.3 & 80.3 & 1.55 & 78.66 & 76.42 & 77.52 & 1.13 \\
\hline House 2 & 84.85 & 80.05 & 82.38 & 40.86 & 85.18 & 85.92 & 85.55 & 1.94 & 73.3 & 93.4 & 82.17 & 0.8 \\
\hline House 6 & 58.64 & 96.32 & 72.76 & 46.41 & 72.85 & 90.4 & 80.68 & 1.22 & 95.09 & 96.07 & 95.58 & 0.19 \\
\hline
\end{tabular}

appliance is not operating alone, model generation will not be successful. Drop in the performance of the HMM as the size of the training set is reduced, is due to the fact that more appliances are not modeled properly and hence are not disaggregated. The training execution time of the proposed method slightly increases as the training set size increases but it is still significantly lower than that of the HMM-based approach for all houses and all training sizes. Indeed, the proposed method needs 32-88 and 1.43-3 times less time for testing than HMM and SVM, respectively, and 40.45-274 and 1.18-6.27 times less time for training than HMM and SVM, respectively.

Table VII present results when random labeling errors are introduced during the training process. For example, $5 \%$ means that every fifth event of one hundred events was randomly labeled during training. Note that it is possible for $F_{M}$ to slightly increase if the number of errors increase due to the decrease in FN and FP. One can see that both methods show robustness to errors in the training dataset.

\section{CONCLUSION}

In this paper we proposed a low-complexity approach for energy disaggregation based on k-means clustering and Support Vector Machine (SVM). Experimental results using REDD data demonstrate the potential of the proposed solutions. Indeed, the proposed approach shows similar performance to that of HMM, with up to 88 and 274 times lower execution time for testing and training, respectively. Tests, conducted by reducing the training size and introducing errors in the training data, showed robustness of the proposed approach, that is capable of performing successful disaggregation using only two days of training data and up to $20 \%$ of errors in the training set.

The applications of such a low-complexity algorithm are 
TABLE V

EXECUTION TIME [SEC] FOR THE THREE REDD HOUSES USING THREE DIFFERENT TRAINING SIZES.

\begin{tabular}{|c|c|c|c|c|c|c|c|c|c|}
\hline \multirow[b]{2}{*}{ House } & \multirow[b]{2}{*}{ trainingsize } & \multicolumn{2}{|c|}{$H M M$} & \multicolumn{2}{|c|}{$k$-means } & \multicolumn{2}{|c|}{$S V M$} & \multicolumn{2}{|c|}{ Proposed method } \\
\hline & & train & test & train & test & train & test & train & test \\
\hline \multirow{3}{*}{ House 1} & 2000 & 15.18 & 21.29 & 0.18 & 0.18 & 0.37 & 0.57 & 0.19 & 0.32 \\
\hline & 5000 & 23.79 & 19.27 & 0.25 & 0.25 & 0.76 & 0.65 & 0.27 & 0.25 \\
\hline & 7000 & 28.32 & 22.90 & 0.2 & 0.2 & 0.83 & 0.78 & 0.7 & 0.26 \\
\hline \multirow{3}{*}{ House 2} & 2000 & 18.38 & 18.56 & 0.09 & 0.25 & 0.43 & 0.72 & 0.1 & 0.32 \\
\hline & 5000 & 21.13 & 18.03 & 0.06 & 0.2 & 0.84 & 0.72 & 0.3 & 0.34 \\
\hline & 7000 & 22.77 & 18.09 & 0.23 & 0.23 & 1.15 & 0.79 & 0.25 & 0.55 \\
\hline \multirow{3}{*}{ House 6} & 2000 & 20.52 & 10.99 & 0.07 & 0.18 & 0.33 & 0.35 & 0.12 & 0.2 \\
\hline & 5000 & 22.46 & 13.91 & 0.09 & 0.14 & 0.56 & 0.45 & 0.15 & 0.26 \\
\hline & 7000 & 30.22 & 16.19 & 0.24 & 0.24 & 0.69 & 0.53 & 0.11 & 0.28 \\
\hline
\end{tabular}

TABLE VI

F-MEASURE (\%) FOR THE TWO NALM METHODS FOR THE REDD HOUSE 2 USING THREE DIFFERENT TRAINING SIZES.

\begin{tabular}{|c||c|c|c||c|c|c|}
\hline \multicolumn{1}{|c||}{} & \multicolumn{3}{c||}{ HMM Proposed combined method } \\
\hline Appliances & 7000 & 5000 & 2000 & 7000 & 5000 & 2000 \\
\hline \hline Refrigerator & 87.69 & 83.42 & 83.55 & 94.35 & 91.88 & 90.22 \\
\hline Microwave & 44.25 & 44.25 & 44.25 & 25.91 & 0 & 0 \\
\hline Dish Washer & 12.32 & 12.32 & 0 & 29 & 0 & 0 \\
\hline Toaster & 64.90 & 64.90 & 0 & 79.13 & 17.1 & 60 \\
\hline Stove & 48.48 & 0 & 0 & 44.4 & 0 & 0 \\
\hline
\end{tabular}

TABLE VII

F-MEASURE (\%) FOR THE TWO NALM METHODS FOR THE REDD HOUSE 2 WHEN ERRORS ARE INTRODUCED IN THE TRAINING SET DATA.

\begin{tabular}{|c||c|c|c|c||c|c|c|c|}
\hline \multicolumn{1}{|c||}{} & \multicolumn{4}{c||}{ HMM } & \multicolumn{3}{c|}{ Proposed combined method } \\
\hline Appliances & $0 \%$ & $5 \%$ & $15 \%$ & $20 \%$ & $0 \%$ & $5 \%$ & $15 \%$ & $20 \%$ \\
\hline \hline Refrigerator & 87.69 & 83.42 & 83.42 & 83.55 & 94.35 & 91.88 & 93.32 & 91.9 \\
\hline Microwave & 44.25 & 44.25 & 44.25 & 0 & 25.91 & 0 & 0 & 0 \\
\hline Dish Washer & 12.32 & 12.32 & 12.32 & 12.32 & 29.2 & 28.57 & 33.33 & 21.4 \\
\hline Toaster & 64.90 & 64.90 & 46.97 & 46.97 & 79.13 & 11.42 & 11.94 & 8.9 \\
\hline Stove & 48.48 & 48.48 & 18.65 & 18.65 & 44.4 & 0 & 0.00 & 0.00 \\
\hline
\end{tabular}

real-time applications of NALM for emerging services such as home automation and intelligent appliance scheduling as well as forming home area networks of virtual power sensor nodes facilitating demand side management. A downside of the proposed approach is that it requires re-training whenever a new appliance is introduced. A part of the future work is developing efficient training methods and detailed evaluation of the system in a real-world environment.

\section{ACKNOWLEDGMENT}

This work is supported in part by the UK Engineering and Physical Sciences Research Council (EPSRC) projects REFIT EP/K002708 and APAtSCHE EP/K002368, under the Transforming Energy Demand in Buildings through Digital 
Innovation (BuildTEDDI) funding programme. The authors would like to thank O. Parson for sharing his code, and J. Kolter and M. Johnson for making the REDD database available.

\section{REFERENCES}

[1] Government response to the consultation on the second version of the Smart Metering Equipment Technical Specifications: Part 2, Department of Energy \& Climate Change UK, December 2013.

[2] G. W. Hart, "Nonintrusive Appliance Load Data Acquisition Method", MIT Energy Laboratory Technical Report, Sept. 1984.

[3] K.C. Armel, A. Gupta, G. Shrimali, and A. Albert, "Is disaggregation the holy grail of energy efficiency? The case of electricity," Energy Policy, vol. 52, pp. 213-234, 2013.

[4] M. Zeifman and K. Roth, "Nonintrusive appliance load monitoring: Review and outlook," IEEE Trans. Consumer Electronics, vol. 57, no. 1, pp. 76-84, Feb. 2011.

[5] A. Zoha, A. Gluhak, M.A. Imran, and S. Rajasegarar, "Non-intrusive load monitoring approaches for disaggregated energy sensing: A survey," Sensors, vol. 12, pp. 16838-16866, Dec. 2012.

[6] S. Barker, S. Kalra, D. Irwin, and P. Shenoy, "NILM redux: The case for emphasizing applications over accuracy," NILM-2014 Workshop, Austin, TX, June 2014

[7] T. Zia, D. Bruckner, and A. Zaidi, "A Hidden Markov Model based procedure for identifying household electric loads," In Proc. IECON2011 37th Annual Conf. IEEE Industrial Electronics Society, Melbourne, Australia, Nov. 2011.

[8] H. Kim, M. Marwah, M. Arlitt, G. Lyon, and J. Han, "Unsupervised disaggregation of low frequency power measurements," in Proc. 11th SIAM Int. Conf. Data Mining, Mesa, AZ, April 2011.

[9] O. Parson, S. Ghosh, M. Weal, and A. Rogers, "Non-intrusive load monitoring using prior models of general appliance types," in Proc. the 26th Conf. Artificial Intelligence (AAAI-12), Toronto, CA, pp. 356-362, July 2012.

[10] J. Kolter, and T. Jaakkola, "Approximate inference in additive factorial HMMs with application to energy disaggregation," in J. Machine Learning, vol. 22, pp. 1472-1482, 2012.

[11] M.J. Johnson and A.S. Willsky, "Bayesian nonparametric Hidden SemiMarkov Models," J. Machine Learning Research (JMLR), vol. 14, pp. 673701, Feb. 2013.

[12] J. Wang, X. Wu, and C. Zhang, "Support vector machines based on K-means clustering for real-time business intelligence systems," Int. J. Business Intelligence and Data Mining, vol. 1, no. 1, pp.54-64, 2005.

[13] Y. Yao, Y. Liu, Y. Yu, H. Xu, W. Lv, Z. Li, and X. Chen, "K-SVM: An Effective SVM Algorithm Based on K-means Clustering," J. Computers, vol. 8, Oct. 2013.

[14] Q. Gu and J. Jan, "Clustered Support Vector Machines," in Proc. AISTATS-2013 16th Int. Conf. Artifical Intelligenece and Statistics, Scottsdale, AZ, 2013

[15] J. Kolter, and M. Johnson, "REDD: A public data set for energy disaggregation research," in Workshop on Data Mining Applications in Sustainability (SIGKDD), San Diego, CA, 2011.

[16] A. G. Ruzzelli, C. Nicolas, A. Schoofs, and G.M.P. O'Hare, "Realtime recognition and profiling of appliances through a single electricity sensor, "IEEE SECON-2010 7th Annual Conf. Sensor Mesh and Ad Hoc Communications and Networks, pp.1-9, Boston, June 2010.

[17] C. Laughman, K. Lee, R. Cox, S. Shaw, S. Leeb, L. Norford, and P. Armstrong, "Power signature analysis," IEEE Power and Energy Magazine, vol. 1, pp 56-63, Mar-Apr 2003.

[18] J. Liang, S. K. K. Ng, G. Kendall, J. W. M. Cheng, "Load signature study part I: Basic concept, structure, and methodology," IEEE Trans. Power Delivery, vol. 25, no. 2, pp. 551-560, Apr. 2010.

[19] M. Berges, E. Goldman, H. S. Matthews, and L. Soibelman, "Learning systems for electric consumption of buildings," in Proc. 2009 ASCE Int. Workshop Computing in Civil Engineering, Austin, TX, 2009.

[20] Electric Power Research Institute, Low-cost NIALMS technology. Electric Power Research Institute, Technical Report TR-198918-V1, Sept. 1997.

[21] J. Powers, B. Margossian, B. Smith, "Using a rule-based algorithm to disaggregate end-use load profiles from premise-level data," IEEE Computer Applications in Power, vol. 4, no. 2, pp. 42-47, 1991.
[22] L. Farinaccio and R. Zmeureanu, "Using a pattern recognition approach to disaggregate the total electricity consumption in a house into the major end-uses," Energy \& Building, vol.30, pp. 245-259, 1999.

[23] M. L. Marceau and R. Zmeureanu., "Nonintrusive load disaggregation computer program to estimate the energy consumption of major end uses in residential buildings," Energy Conversion \& Management, vol. 41, pp. 1389-1403, 2000.

[24] M. Baranski and J. Voss, "Genetic algorithm for pattern detection in NIALM systems," in Proc. SMC-2004 IEEE Int. Conf. Sys., Man and Cybernetics, pp. 3462-3468, Hague, Netherlands, Oct. 2004.

[25] J. Liao, G. Elafoudi, L. Stankovic, and V. Stankovic, "Power disaggregation for low-sampling rate data," 2nd Int. Non-intrusive Appliance Load Monitoring Workshop, Austin, TX, June 2014.

[26] J. Liao, G. Elafoudi, L. Stankovic, and V. Stankovic, "Non-intrusive appliance load monitoring using low-resolution smart meter data," SmartGridComm IEEE International Conference on Smart Grid Communications, Venice, Italy, November 2014.

[27] S. Markonin, I.V. Bajic, and F. Popowich, "Efficient sparse matric processing for nonintrusive load monitoring," 2nd Int. Non-intrusive Appliance Load Monitoring Workshop, Austin, TX, June 2014.

[28] X.L. Xia, M.R. Lyu, T.M. Lok, and G.B. Huang, "Methods of decreasing the number of support vectors via k-mean clustering," in ICIC 2005, LNCS 3644, pp. 717-726, Spinger-Verlag Berlin Heidelberg, 2005.

[29] D.L. Olson and D. Delen, Advanced Data Mining Techniques, Springer, 2008. 